\title{
Analysis of modified degree six chordal rings and traditional chordal rings degree six interconnection network
}

\begin{abstract}
Chordal rings are attractive network interconnection due to their short diameters especially when the degree was increased. Chordal rings were related to find the shortest path in a ring structured. This paper presents an analysis of modified degree six chordal rings (CHRm6) and chordal rings degree six (CR6) or commonly known as traditional chordal rings degree six (CR6). This analysis includes the structures, tree visualization, paths, formulations and geometrical representation. The comparison of diameters was made between theoretical and ideal graphs for these two methods. We tested and compared for a large number of nodes. CHRm6 gives shortest diameters for even and odd source node.
\end{abstract}

Keyword: Chordal rings; Interconnection networks; CHRm6 